\title{
Producción de plantas hijas a partir de estolones en cinco variedades de fresa (Fragaria spp.) manejadas
} bajo condiciones de invernadero en Molinopampa, Amazonas

\section{Production of daughter plants from stells in five strawberry varieties (Fragaria spp.) handled under greenhouse conditions in Molinopampa, Amazonas}

\author{
Mario Oliva ${ }^{1}$ y José Oliva1 ${ }^{1 *}$
}

\section{RESUMEN}

El presente trabajo de investigación fue realizado con el objetivo de evaluar la tasa potencial de propagación de cinco variedades de fresa (Fragaria spp.), bajo las condiciones de invernadero en el distrito de Molinopampa, Amazonas. Las variedades utilizadas fueron Aromas, Camarosa, Albión, Monterrey y San Andreas, las cuales se establecieron en un diseño experimental completos al azar. Como material de genético se utilizaron plantas madres provenientes del INIA Estación Experimental Agraria Donoso y se instalaron en módulos verticales a distanciamiento de $35 \mathrm{~cm}$ entre plantas. El manejo de las plantas se realizó mediante control fitosanitario, fertilización foliar, podas de hojas, deshierbo manual, suministro de riego y aplicación de técnica de emasculación. La respuesta de las variables número de coronas por planta, altura de planta, número de hojas evaluadas en plantas madres tuvieron un comportamiento similar para las cinco variedades de fresa. Una respuesta similar se determinó en la variable longitud de estolón. Sin embargo la variables número de estolones, número de yemas por estolón y número de plantas hijas por planta madre difirieron estadísticamente entre las variedades. Para la producción de plantas hijas mostraron mayor respuesta las variedades monterrey con 26,63 hijas y albión con 26,44 hijas por planta madre.

Palabras claves: Variedad, estolón, plantas madre, yemas, planta hija.

\begin{abstract}
The present research work was carried out with the objective of evaluating the potential propagation rate of five strawberry varieties (Fragaria spp.), under greenhouse conditions in the district of Molinopampa, Amazonas. The varieties used were Aromas, Camarosa, Albión, Monterrey and San Andreas, which were established in a randomized complete experimental design. As a genetic material, mother plants from the INIA Agricultural Experimental Station Donoso were used and installed in vertical modules at a distance of $35 \mathrm{~cm}$ between plants. Plant management was carried out through phytosanitary control, foliar fertilization, leaf pruning, manual weeding, irrigation supply and application of emasculation technique. The response of the variables number of crowns per plant, height of plant, number of leaves evaluated in mother plants had a similar behavior for the five strawberry varieties. A similar response was determined in the stolon length variable. However, the variables number of stolons, number of buds per stolon and number of daughter plants per mother plant differed statistically among the varieties. For the production of daughter plants, the Monterey variety showed a higher response with 26.63 daughters and albion with 26.44 daughters per mother plant.
\end{abstract}

Keywords: Variety, stolon, mother plants, buds, daughter plant.

\footnotetext{
${ }^{1}$ Asociación de Productores Conservacionistas de Molinopampa - APROCOM. Av. Libertad N 734, Chachapoyas, Perú

"Autor de correspondencia. E-mail: agroliva.123@hotmail.com
} 


\section{INTRODUCCIÓN}

La fresa es una especie vegetal, cuyo seudofruto es consumido como fruto fresco por la humanidad desde épocas muy antiguas, cuyo fruto es caracterizado por su agradable aroma y sabor (Sánchez-Sánchez, 2006). Antes del descubrimiento del continente Americano la especie más utilizada fue Fragaria vesca; sin embargo en la actualidad se utilizan híbridos interespecíficos, principalmente cruces artificiales entre las especies Fragaria chiloencis y Fragaria virginiata. Un pequeño grupo de estas variedades híbridas como Oso Grande, Chandler, Camarosa, Aromas, Sugar Baby y Monterrey fueron introducidas a Sudamérica y especialmente por las zonas costeras del país. En el Perú, alrededor del $90 \%$ de la producción de fresa se encuentra en el departamento de Lima, el resto dela producción está distribuida en el interior del país a través de pequeños agricultores que disponen de pequeñas áreas de cultivo de fresa con variedades poco conocidas por ellos mismos, y cuyo consumo es principalmente en fresco (Laguna et al., 2006).

En la fase de cultivo, los agricultores establecen en los meses de julio o inicio de agosto, realizando la cosecha en los meses de enero a abril. En el mes de abril seleccionan las coronas y las establecen en bancales de 30 $\mathrm{cm}$ de alto por $1 \mathrm{~m}$ de ancho, a una densidad de 100 coronas por $\mathrm{m}^{2}$. Dicho material es el que utilizan como semilla para establecer el cultivo en periodo agrícola siguiente (Benavides et al., 2007). Sin embargo, los agricultores han observado una significativa disminución del rendimiento potencial de las variedades. Por otro lado se han observado que ciertas plantas de ambas variedades desarrollan estolones durante el desarrollo del cultivo; los cuales se eliminan para que estos no afecten la producción y el tamaño de los frutos. A pesar de la merma de los rendimientos los agricultores continúan sembrando fresa.

La fresa es un vegetal que puede vivir por mucho tiempo; sin embargo, se mantiene en producción económicamente rentable, durante los primeros dos años. En plantaciones de mayor edad, las plantas se muestran débiles, con bajo rendimiento y frutos de menor cali- dad, debido a una mayor incidencia de plagas y enfermedades. Por ser una planta híbrida, no se utilizan sus semillas para propagarla. Su sistema de crecimiento y formación de nuevas coronas y estolones permite una propagación vegetativa rápida y segura. Esta planta normalmente se propaga por estolones, obtenidos de plantas madres que han estado sometidas a largos períodos de frigoconservación, característica que estimula un gran crecimiento vegetativo cuando son llevadas al campo (Barrera y Sánchez 2003).

A partir de la década de los 50's, los productores de fresa han estado condicionados en la adquisición de plantas "madre" de fresas provenientes de los Estados Unidos (López et al., 2014), entre las que destacan: Festival, Camino Real, Sweet Charly, Camarosa, y Albion. El productor adquiere la planta "madre" en cada ciclo a través de un intermediario o directamente de Ekland Marketing o Euro Semillas S.A. de C. V. pagando USD $\$ 500$ a 1000 dólares por millar de plantas según la variedad, más regalías que en el periodo 2001 a 2005 aumentaron hasta 300\%, así como trámites de importación, transporte, entre otros, lo cual genera una disminución en la rentabilidad del cultivo. Estudios comparativos sobre la calidad de la fruta de las variedades mexicanas desarrolladas para Michoacán, CP Roxana, CP Paola con las variedades extranjeras Aromas, Camarosa y Festival, reportan adecuada calidad de las variedades nacionales; sin embargo, las variedades nacionales requieren un manejo cuidadoso en pos cosecha debido a su menor firmeza. Asimismo, estudios sobre la calidad de propagación de las variedades Zamorana, Jacona y las variedades extranjeras Festival y Albion en viveros de Michoacán, reportaron que las variedades mexicanas son altamente sensibles a las condiciones de altitud en las que se establecen los viveros, generando una menor capacidad de producción de estolones y plantas "hijas" en comparación con la variedad Festival, aunque superan al genotipo Albion en la producción de vivero (Bautista et al., 2012).

La competitividad de la producción agrícola depende principalmente de la organización y compromiso de 

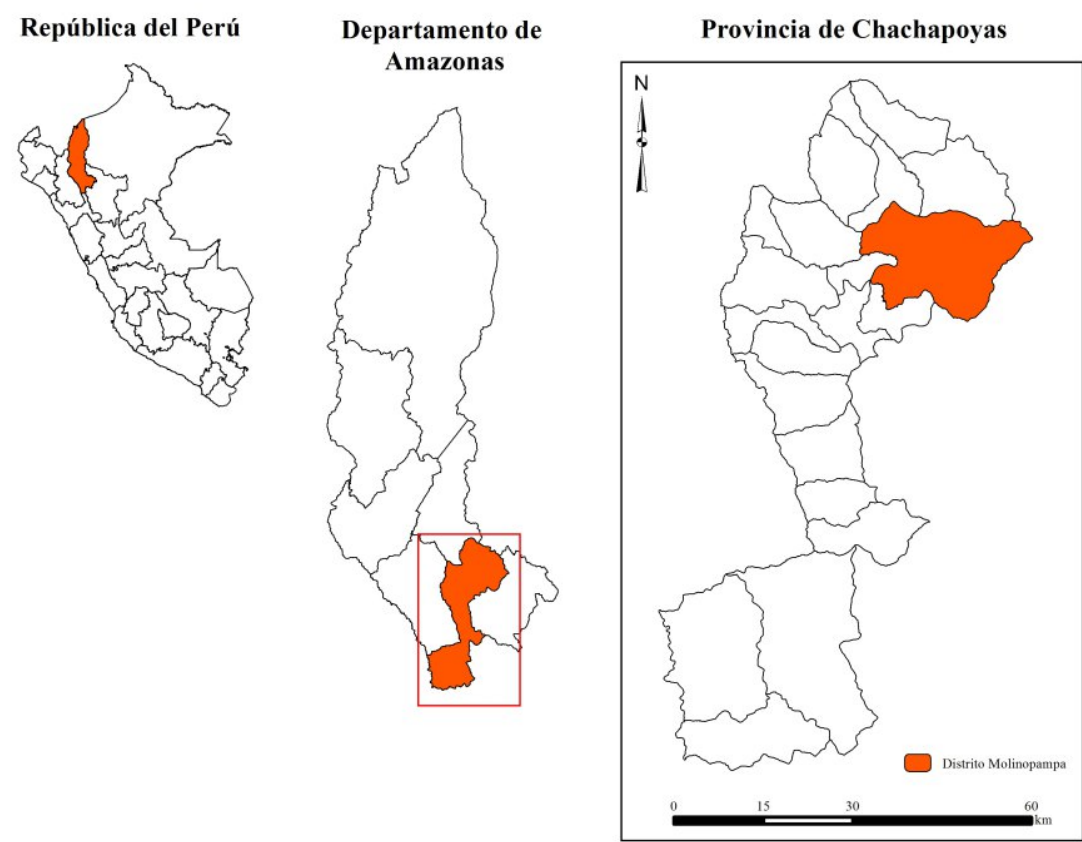

Figura 1. Ubicación del área de estudio en el distrito de Molinopampa (provincia de Chachapoyas)

los productores para el fortalecimiento de la cadena agroalimentaria de la fresa. Consecuentemente, es urgente la actualización de un plan rector del sistema "producto fresa" estatal que sea validado con base en las demandas del mercado, el reconocimiento y participación a nivel regional y nacional de los productores y otros agentes con un fin común (Castillejo, 2011). En este contexto el objetivo del presente estudio fue determinar la producción de plantas hijas por cada planta madre instalada mediante propagación por estolones en cinco variedades de fresa en la búsqueda de mejorar la calidad de la semilla.

\section{MATERIALES Y MÉTODOS}

\section{Ubicación del estudio}

El estudio en su totalidad se realizó en el invernadero de aclimatación de plantas madre de variedades de fresa, ubicado en el anexo de Espadilla, distrito de Molinopmapa durante los meses de junio y diciembre del año 2017. Su ubicación georeferencial responde a 18M 0216327, UTM 9306478, con temperatura promedio anual de $15,5^{\circ} \mathrm{C}$, precipitación anual entre 1480 y $1530 \mathrm{~mm}$ y una altitud de 2235 m.s.n.m. (Figura 1).

\section{Instalación de invernadero de aclimatación}

El invernadero tipo multitúnel fue construido en un área total de $80 \mathrm{~m}^{2}$ utilizando estructura de tubo galva- nizado de 3/4" de diámetro con una altura total de 2,90 metros, siendo de 4 metros de ancho y 10 metros de fondo cada túnel. La cobertura del invernadero consta de plástico transparente agrofilm extendida en forma de techo y malla antiáfida utilizada en la parte vertical desde el piso hasta una altura de 1,50 metros, punto de inicio del arco metálico de cobertura plástica. En tanto el piso del invernadero fue afirmado con piedra chancada caliza extendida y en el interior del invernadero fue instalado un lavadero para desarrollar trabajos de propagación vegetativa.

El invernadero se encuentra debidamente iluminado con dos fluorescentes distribuidos en cada túnel y con conexiones para uso de equipos eléctricos como bomba de succión y otros. Del mismo modo, se tiene instalado sistemas de riego tanto por goteo (suministro de agua a cada planta instalada) y riego por nebulización aérea distribuido en todo el ambiente interno del invernadero. En cuanto a la infraestructura para la producción de estolones, se ha instalado un total de diez módulos de tipo piramidal, soportando a seis contenedores horizontales de madera aserrada y cada módulo con una capacidad para instalación de 42 plantas madres de fresas.

\section{Material genético}

En el presente estudio se utilizaron como material 
genético un total de 420 plantas madres procedentes del Instituto de Innovación Agraria (INIA), Estación Experimental Agraria Donoso ubicado en la provincia de Huaral, departamento de Lima. El total de plantas madres estuvieron distribuidas en cinco variedades de fresa (aromas, camarosa, albión, monterrey y san andreas), con 84 plantas por cada variedad. Las plantas fueron transportadas vía terrestre desde la ciudad de Lima hasta Molinopampa (invernadero de manejo) a raiz desnuda envueltas en papel periódico y embaladas cajas de cartón con orificios para respiración.

\section{Diseño experimental}

Para realizar la evaluación de las plantas madres en condiciones de invernadero, se utilizó un Diseño Completo al Azar (DCA). Se establecieron las cinco variedades como tratamientos de estudio T1 (aromas), T2 (camarosa), T3 (albión), T4 (monterrey) y T5 (san andreas) con cinco repeticiones por tratamiento, cada unidad muestral estuvo constituida por cinco plantas madres, resultando un total de 125 plantas madres de fresa sometidas a evaluación.

\section{Instalación de plantas madres}

Previo a la instalación de las plantas madres, se preparó el sustrato compuesto por tierra agrícola, tierra de bosque y arena (con proporción de 2-1-1 respectivamente) se adicionó abono orgánico en base a NPK y cantidad moderada de cal agrícola como regulador de $\mathrm{pH}$ del suelo, el sustrato preparado fue humedecido y llenado en los contenedores horizontales de los módulos de producción, luego este fue cubierto con fibra de coco preparado con el fin de amortiguar las gotas de agua de riego e incorporación de abono orgánico al suelo a través de la descomposición. Los contenedores de sustrato fueron recubiertos interna y externamente con plástico blanco con orificios distanciados para colocar las plantas. Luego se procedió a eliminar el $70 \%$ del follaje de las plantas, se sumergió la raíz en una solución con enraizante y finalmente se procedió a instalar un total de 420 plantas madres de fresa en 10 módulos, con 84 plantas por cada variedad con distanciamiento de siembra de $35 \mathrm{~cm}$ entre plantas.

\section{Manejo de plantas instaladas}

Según la necesidad y reqierimiento de las plantas se proporcionó agua a las plantas mediante sistema de riego por goteo suministrando gotas de agua directamente al sustrato alrededor de cada planta madre instalada, asimismo para proveer de humedad al follaje de las plantas se utilizó el sistema de riego por nebulización aérea, aplicando una frecuencia de riego según la necesidad de las plantas para aproximar la capacidad de campo. En tanto, el control de malezas en las plantas se realizó de forma manual y permanente, eliminando ciertas malezas aparecidas en partes descubiertas junto a las plantas de fresa. Se procedió con control preventivo de las plantas contra insectos chupadores y desfoliadores, para lo cual se utilizó insecticida comercial aplicando en cantidades permitidas, asimismo se aplicó fertilizante foliar en proporción 20-20-20 inicialmente cada 15 días y luego de forma mensual en algunas ocasiones mezclado con fungicida en polvo para prevenir la proliferación de hongos.

A partir de 3 meses de crecimiento las plantas fueron sometidas a la técnica de emasculación vegetal, práctica que consistió en la extracción de las flores con la ayuda de tijera de podar y así evitar la formación y consecuentemente la producción de frutos. Al cortar las flores, simultáneamente se induce a la generación de brazos laterales, lo que se denomina la producción de estolones y en cada nudo a lo largo de los estolones se generan las yemas y a partir de estas se generan las plantas hijas de las plantas madres instaladas en el invernadero.

\section{Toma de datos}

Durante el periodo de establecido el cultivo se realizaron permanentemente la eliminación de botones florales, hojas amarillentas y dañadas, limpieza constante del follaje de las plantas madres. La toma de datos de todas las variables en estudio se realizó durante los meses de junio y diciembre del 2017, periodo en el cual las plantas alcanzaron la mayor productividad de estolones y consecuentemente mayor cantidad de plantas hijas. 


\section{Variables evaluadas}

En el periodo de trabajo se evaluaron diversas variables como: nivel de sobrevivencia de plantas madres instaladas, número de coronas por planta, altura de planta, número promedio de hojas, número de estolones por planta, longitud de estolón, número de yemas por estolón y número de hijas por planta.

\section{Procesamiento de datos y análisis estadístico}

Los datos fueron capturados en una hoja electrónica de evaluación para su posterior análisis estadístico mediante el software estadístico SPSS Versión 23, los datos fueron sometidos a un análisis de varianza
(ANOVA) y prueba de comparación múltiple Tukey al $5 \%$.

\section{RESULTADOS}

La evaluación de sobrevivencia de plantas instaladas en invernadero, determinó un $100 \%$ de sobrevivencia de plantas madres para las cinco variedades en estudio. Mediante análisis estadístico se determinó que no hubo efecto significativo de las cinco variedades de fresas en estudio sobre las variables número de coronas, altura de planta y número de hojas, observándose un solo rango "a" (Tabla 1).

Tabla 1. Comparación de valores medios de las principales variables de plantas madres

\begin{tabular}{|c|c|c|c|c|}
\hline Factor & Niveles & $\mathbf{N}^{\circ}$ de coronas & $\begin{array}{c}\text { Altura planta } \\
\text { (cm) }\end{array}$ & $\mathbf{N}^{\circ}$ hojas \\
\hline \multirow{5}{*}{ Variedad de fresa } & Aromas & $3,24^{\mathrm{a}}$ & $18,64^{\mathrm{a}}$ & $22,24^{\mathrm{a}}$ \\
\hline & Camarosa & $3,32^{\mathbf{a}}$ & $18,52^{a}$ & $22,52^{a}$ \\
\hline & Albión & $3,28^{a}$ & $20,02^{\mathbf{a}}$ & $22,36^{a}$ \\
\hline & Monterrey & $3,36^{\mathbf{a}}$ & $20,04^{\mathrm{a}}$ & $22,64^{\mathrm{a}}$ \\
\hline & San andreas & $3,28^{\mathrm{a}}$ & $19,68^{\mathbf{a}}$ & $22,40^{\mathrm{a}}$ \\
\hline
\end{tabular}

\section{Producción de estolones}

El rendimiento en la producción de estolones en el cultivo de fresa indicó diferencias estadísticas significativas entre tratamientos a través de la prueba de Tukey. En la tabla 2 se puede observar que el mayor promedio en producción de estolones se logró con los tratamientos T3 y T4 con 8,80 y 8,76 estolones por planta respectivamente. El menor promedio se obtuvo en el tratamiento T5 con una producción de 8,12 estolones por planta madre.

Tabla 2. Prueba de Tukey para producción de estolones

\begin{tabular}{ccc}
\hline Tratamiento & $\begin{array}{c}\text { Númerode } \\
\text { estolones }\end{array}$ & $\begin{array}{c}\text { Pruebade } \\
\text { Tukey }\end{array}$ \\
\hline T1 & 8,20 & $\mathrm{BC}$ \\
T2 & 8,16 & $\mathrm{BC}$ \\
T3 & 8,80 & $\mathrm{~A}$ \\
T4 & 8,76 & $\mathrm{~A}$ \\
T5 & 8,12 & $\mathrm{C}$ \\
\hline
\end{tabular}

Se observó que las variedades de fresa albión y monterrey obtuvieron mayor respuesta en la producción de estolones en invernadero bajo las condiciones ambientales de Molinopampa, tal como se aprecia en la figura 2 .

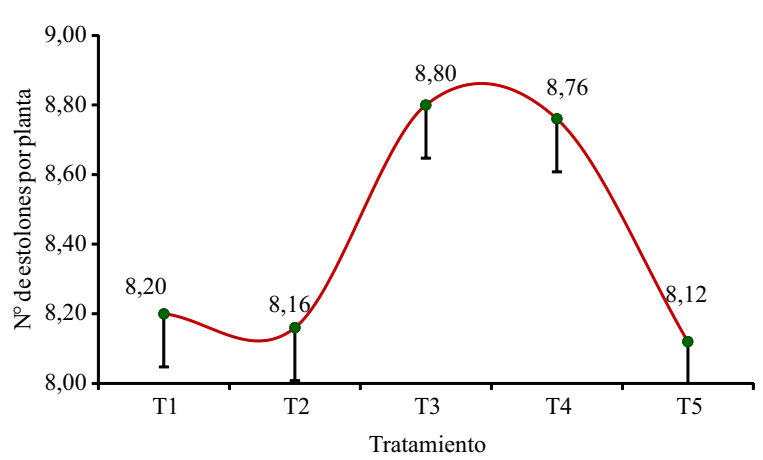

Figura 2. Número de estolones por planta en las variedades de fresa.

\section{Longitud de estolones}

La longitud de estolones en el cultivo de fresa mostró que no existieron diferencias estadísticas significativas entre tratamientos a través de la prueba de Tukey. En la tabla 3 se puede observar que el mayor promedio en longitud de estolones se logró con el tratamiento T1 con $60,52 \mathrm{~cm}$. El menor promedio se obtuvo en el tratamiento $\mathrm{T} 2$ con $59,35 \mathrm{~cm}$ de longitud en estolón.

Tabla 2. Prueba de Tukey para longitud de estolones

\begin{tabular}{ccc}
\hline Tratamiento & $\begin{array}{c}\text { Longitud de } \\
\text { estolones }\end{array}$ & $\begin{array}{c}\text { Prueba de } \\
\text { Tukey }\end{array}$ \\
\hline T1 & 60,52 & $\mathrm{~A}$ \\
T2 & 59,35 & $\mathrm{~A}$ \\
T3 & 60,18 & $\mathrm{~A}$ \\
T4 & 60,24 & $\mathrm{~A}$ \\
T5 & 60,06 & $\mathrm{~A}$ \\
\hline
\end{tabular}


Se logró concluir que la variedad de fresa aromas obtuvo mayor longitud en estolones en plantas madres manejadas bajo las condiciones de invernadero, escenario que se aprecia en la figura 3.

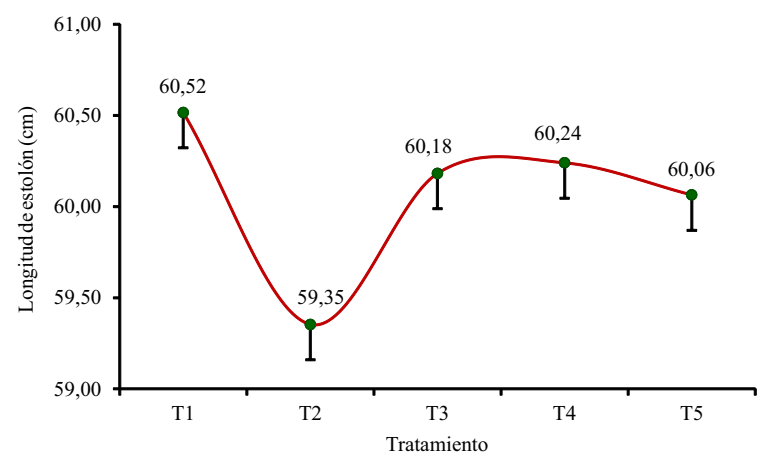

Figura 3. Longitud del estolón en las variedades de fresa.

\section{Desarrollo de yemas por estolón}

En cuanto a la formación de yemas a lo largo de los estolones se establecieron diferencias estadísticas altamente significativas entre tratamientos mediante la prueba Tukey. En la tabla 4 se puede observar que el mayor promedio de número de yemas por estolón se presentó en los tratamientos T4 y T3 con 3,04 yemas y 3,00 yemas respectivamente. En contraste el menor promedio se obtuvo con el tratamiento T5 con 2,48 yemas por estolón

Tabla 4. Prueba de Tukey para formación de yemas

\begin{tabular}{ccc}
\hline Tratamiento & $\begin{array}{c}\text { Númerode } \\
\text { yemas }\end{array}$ & $\begin{array}{c}\text { Pruebade } \\
\text { Tukey }\end{array}$ \\
\hline T1 & 2,52 & $\mathrm{~B}$ \\
T2 & 2,60 & $\mathrm{~B}$ \\
T3 & 3,00 & $\mathrm{~A}$ \\
T4 & 3,04 & $\mathrm{~A}$ \\
T5 & 2,48 & $\mathrm{~B}$ \\
\hline
\end{tabular}

Durante el periodo de evaluación se observó que las variedades monterrey y albión obtuvieron los mejores promedios en formación de yemas por estolón, tal como se puede apreciar en la figura 4.

\section{Producción de plantas hijas}

La producción de plantas hijas mostró diferencias estadísticas significativas entre tratamientos mediante la prueba de Tukey. En la tabla 5 se puede observar que el mayor promedio en la producción de plantas hijas se presentó en los tratamientos T4 y T3 con 26,63 hijas y 26,44 hijas respectivamente.

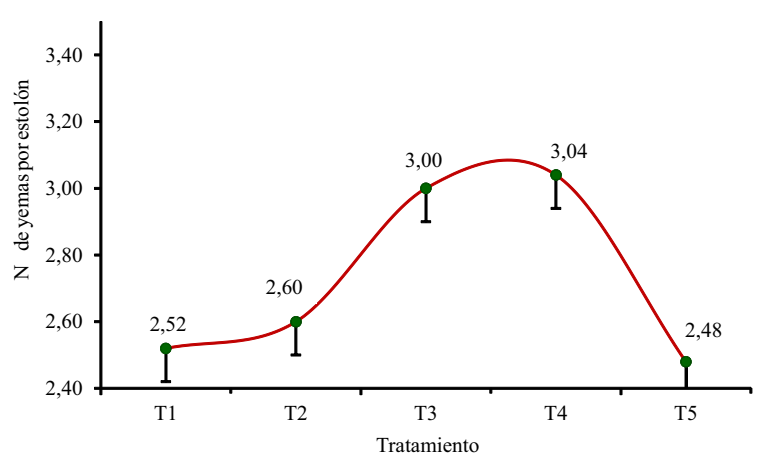

Figura 4. Número de yemas por estolón en las variedades de fresa.

Tabla 5. Prueba de Tukey para la producción de hijas

\begin{tabular}{ccc}
\hline Tratamiento & $\begin{array}{c}\text { No dehijas }_{\text {pormadre }} \\
\text { Prueba de } \\
\text { Tukey }\end{array}$ \\
\hline T1 & 20,67 & $\mathrm{~B}$ \\
T2 & 21,22 & $\mathrm{~B}$ \\
T3 & 26,44 & $\mathrm{~A}$ \\
T4 & 26,63 & $\mathrm{~A}$ \\
T5 & 20,17 & $\mathrm{~B}$ \\
\hline
\end{tabular}

En el periodo evaluado se observó que las variedades monterrey y albión obtuvieron mejor promedio en la producción de número de hijas a partir de plantas madres instaladas en el invernadero, según se aprecia en la figura 5.

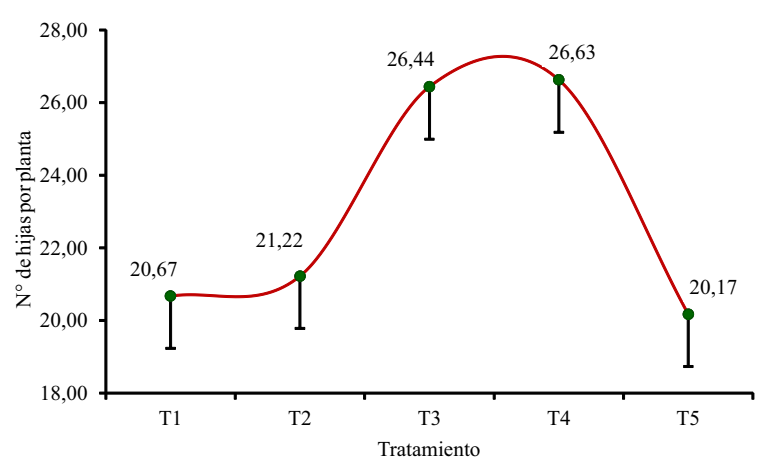

Figura 5. Número de plantas hijas en las variedades de fresa

\section{DISCUSIÓN}

Una planta de fresa de buena calidad debe tener raíces abundantes, coronas múltiples, yemas diferenciadas y alto contenido de carbohidratos para establecerse rápidamente en el terreno de cultivo, y con ello obtener una producción precozy de alto rendimiento de estolones como la base para la producción vegetativa de plantas hijas (Tworkoski et al., 2001). Las variedades de fresa están relacionadas con la temperatura y el fotoperiodo, ya que las altas temperaturas, en condiciones de vivero, afectan la calidad de las plantas hijas. 
Según Larson (2000), la producción de flores y frutos, así como la producción de coronas y estolones son caracteres que si bien están determinados por el genotipo de fresa, estos son muy influenciados por los factores ambientales como por la altura donde se instalan las plantas, temperatura de la zona, luz solar y estado nutricional del suelo. La altitud afecta el tamaño de la corona de las plantas y el número de coronas. El establecimiento de viveros de fresa en altas altitudes, puede provocar daños a las plantas por las bajas temperaturas, en contraste, en bajas altitudes las plantas llegan a presentar menor contenido de almidón, con un bajo rendimiento; por otro lado, se puede obtener mayor número de coronas en periodos más cortos, en comparación con las plantas producidas en elevaciones altas (Monroy et al., 2002).

Toledo (2003) señala que la distancia de siembra para la producción de estolones de fresa en condiciones de invernadero es de al menos $30 \mathrm{~cm}$ entre plantas, lo cual permite estimular la producción de estolones, en dependencia de la variedad y de otros factores. En tanto, Hernández-Soto et al., (2011) reportan que las plantas madres de fresa bajo condiciones de invernadero producen en promedio de 6.9 estolones por planta.

Toledo (2003) estima que una planta madre puede llegar a producir 40 hijas. Por su parte, HernándezSoto et al., 2011) reporta en su estudio sobre 10 cultivares de fresa, que obtuvo un promedio de 27,4 plantas hijas a partir de una planta madre. (Sánchez-Sánchez, 2006) en su estudio expresa, que si bien la multiplicación por estolones tiene una mayor tasa de propagación que la de coronas, es importante determinar en base al cultivar las mejores condiciones ambientales en la que dicho material se adecue mejor a fin de alcanzar las mayores tasas de producción de hijas a través de los estolones. Al parecer la producción de hijas a partir de estolones será más favorable en los meses del año con mayores temperaturas y a altitudes menores bajo las cuales se cultiva la variedad para la producción de semilla vegetativa.

\section{CONCLUSIONES}

Según los resultados obtenidos en el presente estudio y bajo los efectos de los descriptores a nivel de plantas madre se considera que las variedades albión y monterrey obtuvieron en promedio mayor respuesta en número de coronas por planta, altura de planta y número de hojas por planta madre, aspectos que fueron evidenciados mediante la comparación de valores medios.

Por su parte sobre la respuesta de producción de estolones, formación de yemas y desarrollo de plantas hijas está relacionado directamente con los factores ambientales y del genotipo del cultivar. Las cinco variedades de fresa evaluadas presentaron un comportamiento diferenciado para las variables como capacidad de producción de estolones, formación de yemas y desarrollo de plantas hijas; resaltando de manera favorable las variedades albión y monterrey que obtuvieron un mayor número de plantas hijas como objetivo primordial del presente estudio.

\section{REFERENCIAS BIBLIOGRÁFICAS}

Barrera, G., and C. Sánchez. 2003. Caracterización de La Cadena Agroalimentaria/Agroindustria Nacional, Identificación de Sus Demandas Tecnológicas: Fresa. Morelia (México): Programa Nacional Estratégico de Necesidades de Investigación y de Transferencia de Tecnología

Bautista, G., Z. Calderón, D. Jaen, y A. Curiel. 2012. "Propagation Capacity and Plant Quality of Mexican and Foreign." Revista Chapingo Seria Horticultura 18(1): 113-23.

Benavides, A., J. C. Contreras, y R. L. Miranda. 2007. "Fertilización Orgánica Sobre Tres Genotipos de Fresa (Fragaria spp.) En Las Sabanas, Madríz." La Calera 1:54-558.

Castillejo, L. E. 2011. Aplicación de Azospirillum y Su Efecto En La Calidady Rendimiento de Fresa (Fragaria x Ananassa) Var. Albion Cultivadad En Invernadero. Tesis de maestría. Instituto Politécnico Nacional. Jiquilpan (México). 
Hernández-Soto, D., M. T. Garza-Carranza, y E. Guzmán-Soria. 2011. “Competitividad de La Fresa Mexicana de Exportación a EEUU : Un Modelo de Equilibrio Parcial." Revista Globalización, Competitividady Gobernabilidad $5 \quad(3): \quad 102-14$. doi:10.3232/GCG.2011.V5.N3.06.

Laguna, R., J. Cisne, y A. Benavides. 2006. “Diagnosis y Comportamiento de Enfermedades En Tres Genotipos de Fresa (Fragaria spp.) En Las Sabanas, Madríz." La Calera 1:27-32.

Larson, D. K. 2000. “Comportamiento y Manejo de La Fresa: Desarrollo de Programas Para Máxima Calidad y Rendimiento En México.” En Simposio Internacional de Fresa. Zamora (México).

López, L. L., D. L. A. Guzmán-Ortíz, J. A G. Berumen, C. G. C Marmolejo, y J. J. Peña-Cabriales. 2014. “Consideraciones Para Mejorar La Competitividad de La Región 'El Bajío' En La Producción Nacional de Fresa." Revista Mexicana de Ciencias Agrícolas 5 (4): 673-86.

Monroy, J., J. A. Vera-Núñez, M. A. Carrera, O. A. Grageda-Cabrera, y J. J. Peña-Cabriales. 2002. “Absorción De Nitrógeno (15N) Y Productividad Del Agua Por El Cultivo de Fresa (Frafaria x Ananasa) En 'El Bajio', México." Terra Latinoamericana 20 (1): 65-69.

Sánchez-Sánchez, L. J. 2006. “Producción Orgánica de Fresa (Frafaria x Ananasa), En Tubos de PVC." Mazatlán (México): Universidad Autónoma de Sinaloa.

Toledo, M. 2003. Guía Para La Producción de Fresa En Honduras. La Lima (Honduras): FHIA.

Tworkoski, T. J., T. E. Benassi, y F. Takeda. 2001. “The Effect of Nitrogen on Stolon and Ramet Growth in Four Genotypes of Fragaria Chiloensis L.” Scientia Horticulturae 88: 97-106. doi:10.1016/S0304-4238(00)00198-9. 